\title{
Learning Force Concepts using Visual Trajectory and Haptic Force Information at the Elementary School Level
}

\author{
J. Jay Young ${ }^{1}$, Carolyn Stolfi ${ }^{1}$, Hong Z. Tan ${ }^{1}$, Joël Chevrier ${ }^{2}$, Brien Dick ${ }^{3}$, Gary Bertoline ${ }^{4}$ \\ ${ }^{1}$ Haptic Interface Research Laboratory, Purdue University, West Lafayette, IN, USA \\ ${ }^{2}$ Institut Néel, CNRS/UJF, Grenoble, France \\ ${ }^{3}$ Happy Hollow Elementary School, West Lafayette, IN, USA \\ ${ }^{4}$ Department of Computer Graphics Technology, Purdue University, West Lafayette, IN, USA
}

\begin{abstract}
The present study investigated the use of visual trajectory and haptic force information in learning concepts involving force. Specifically, learning modules for instructing buoyant forces were developed for use with a computer monitor and a force feedback device. Students from an elementary school at the fourth and sixth grades were recruited to participate in the study. The students were separated into visual and visuohaptic groups to measure the possible benefits haptic feedback might provide as compared to the vision-alone condition. A 10-question content test was developed and administered before and after the learning activities. The pretest and posttest scores showed that all students benefited from the computer simulations. The visuohaptic group did not perform significantly better than the visual group. An important finding was that the fourth graders learned as much as the sixth graders, despite their younger age and little prior exposure to concepts such as density and volume, which are important for understanding buoyancy. Future work will design instructional and assessment materials that focus more on the haptic modality.
\end{abstract}

KeYWORDS: Haptics, force concepts, trajectory vs. force information, buoyancy, visuohaptic simulation.

INDEX TERMS: H.5.1 [INFORMATION INTERFACES AND PRESENTATION]: Multimedia Information Systems - Artificial, augmented, and virtual realities; H.5.2 [INFORMATION INTERFACES AND PRESENTATION]: User Interfaces (D.2.2, H.1.2, I.3.6 - Haptic I/O; K.3.1 [COMPUTERS AND EDUCATION]: Computer Uses in Education - Computer-assisted instruction (CAI)

\section{INTRODUCTION}

Haptic perception as a pedagogical strategy for understanding STEM (science, technology, engineering, and mathematics) concepts has been a driving force toward using haptic devices in the classroom. The desire to provide tangible evidence for abstract topics offers potential benefits for making knowledge more accessible and engaging to learners. Recognizing the potential of haptics technology in providing a "hands-on" learning experience

\{youngj, cstolfi, hongtan, bertolig\}@purdue.edu, joel.chevrier@grenoble.cnrs.fr, dickb@wl.k12.in.us

IEEE World Haptics Conference 2011

21-24 June, Istanbul, Turkey

978-1-4577-0297-6/11/\$26.00 @2011 IEEE for students, many researchers have utilized force-feedback devices in teaching dynamics, biology and physics concepts [1-7]. Despite the enthusiastic expectations of the researchers, however, the results have been mixed and "(previous studies) have yet to provide empirical evidence for the existence of a cognitive impact of haptic technology" [8]. While some studies demonstrate the unequivocal benefits of force feedback in training and learning [910], others paint a less than clear-cut picture [11]. It is our view that haptics will have a bigger impact in presenting information that cannot be easily conveyed visually, such as the magnitude and direction of buoyant force. Therefore, the present study investigated the use of visual trajectory and haptic force information in creating learning modules for instructing concepts related to buoyant forces.

We also hypothesized that the use of visuohaptic simulations may be particularly beneficial to younger learners who have yet to develop the mathematical skills to derive force values from visual portrayal of object trajectory. Doing so requires taking the second derivative of trajectory in order to compute force, as illustrated below. Traditionally, force concepts are taught to students as an extrapolation of Newton's Second Law, given by

$$
F=m a
$$

where $F$ is force, $m$ is the mass of the object, and $a$ is the acceleration of the object. This approach assumes that students have an intuitive understanding that acceleration is the second derivative of position:

$$
a=\frac{d^{2} x}{d t^{2}}
$$

where $x$ is the object's position with respect to time. Elementary school students who have yet to be exposed to this degree of formalism, however, must rely on their experiences with forces to create a basis for understanding. This approach exposes the students to a greater potential for error when they are asked to draw from their own intuition.

Visuohaptic simulations can provide learners with an environment in which understanding force concepts is neither rooted in mathematical representation nor dependent on highly contextualized knowledge. Morris et al. used virtual environments as a way to teach participants to learn and recall a sequence of forces [12]. They discovered that participants were able to recall the force patterns best when the information was presented visuohaptically.

Improvements in haptic device design have made haptic devices more affordable and, therefore, more accessible to users. The Falcon 3-DOF force-feedback device has already been used in 
large classroom settings ( $>20$ students) to teach undergraduate students about point-charge interactions [4].

In this study, fourth and sixth grade students are asked to use the Falcons to learn about buoyant forces by using a simulation in which they place cubes with different material properties into tanks filled with liquids of varying density. Buoyancy is a particularly challenging concept for students at all levels to understand due to the difficulties that arise when distinguishing buoyant force from the total net force acting on the object:

$$
F_{t o t}=W+B=\left(V_{s} \rho_{s}-V_{l} \rho_{l}\right) g
$$

where $F_{\text {tot }}$ is the total net force, $W$ is the weight force, $B$ is the buoyancy force, $V_{s}$ is volume of the object, $\rho_{s}$ is the density of the object, $V_{s}$ is the volume of the liquid displaced by the object, $\rho_{l}$ is the density of the surrounding liquid, and $g$ is the acceleration due to gravity. The buoyant force is represented by $-V_{l} \rho_{l} g$ and the weight force is represented by $V_{s} \rho_{s} g$.

In a two-paper series, Loverude et al. demonstrated that undergraduate students had trouble identifying that the buoyant force of an object was directly related to the amount of volume displaced by that object when partially or fully submerged in a liquid [13-14]. The present study was developed to specifically address four of the most common misconceptions identified by Loverude et al. They were object volume, density, trajectory, and surrounding fluid density. In the present study, objects in each simulation were presented as cubes; the activities were designed such that students had the ability to focus on a single object parameter (mass, volume, or density, etc.) without the visual distractions presented by realistic geometries of physical phenomena or the mathematical rigor required by highly analytical thinking.

\section{METHODS}

\subsection{Participants}

Participants were students from the Happy Hollow Elementary School (West Lafayette, IN, USA) at the fourth and sixth grade levels. Eighty-seven participants (twenty-three from fourth grade and sixty-four from sixth grade) took part in the present study. None had previous experience using a haptic device. There were fifteen males and eight females from the fourth grade and thirtyfive males and twenty-nine females from the sixth grade. Written consent was obtained by all participants and their guardians. The Purdue University Institutional Review Board approved all consent forms.

\subsection{Apparatus}

Visual information was displayed on a 19" LCD monitor. Haptic feedback was presented through a Falcon 3-DOF force-feedback device (Novint Technologies, Inc., Albuquerque, NM, USA). The Falcon is a commercially available device that was selected for its affordability and ease of use. Software was developed in $\mathrm{C}++$ using the CHAI3D, OpenGL and ODE libraries.

\subsection{Experimental Conditions}

Participants were divided into two groups after completing a training session using the Falcon devices. The visual (V) group received visual information with no haptic force feedback provided to the user, while the visuohaptic $(\mathrm{VH})$ group received visual information with haptic force feedback provided to the user. The V and $\mathrm{VH}$ groups received the same visual stimuli for the duration of the study.

\subsection{Stimuli}

$\mathrm{V}$ and $\mathrm{VH}$ students completed five activities during the buoyancy session to illustrate the four core ideas most relevant to common misconceptions:

Activity 1:

Activity $2:$

liquid displacement as a representation of an object's volume;

Activity 3: density of an object as a comparison floating/sinking behavior of an object based on the object's mass; between the object's mass and volume;

Activities 4 \& 5: effects of surrounding fluid density on an object's floating/sinking behavior.

All instructional materials were presented through Microsoft PowerPoint. The slides were read aloud while students followed along to introduce each concept prior to allowing the students to participate in an activity (see Fig. 1).

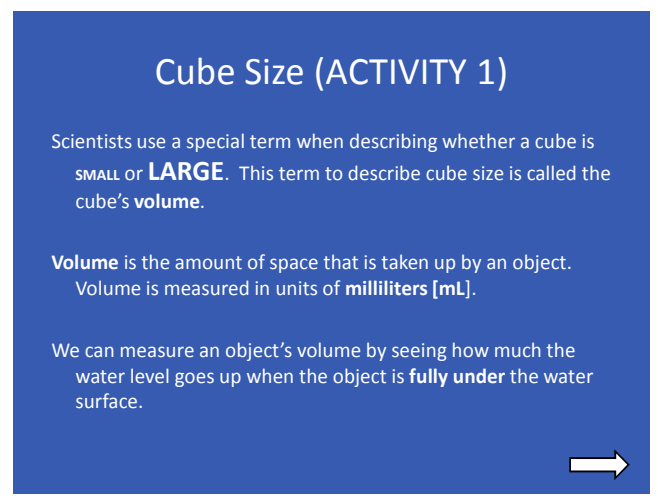

Figure 1. A sample slide of the PowerPoint instructional materials

All hands-on learning activities required the students to interact with visual or visuohaptic simulations in a virtual environment. The students used the Falcon force feedback device either as a 3D mouse (for the $\mathrm{V}$ group where force output from the Falcon was disabled) or as both a 3D mouse and a force display (for the $\mathrm{VH}$ group). The visual display always consisted of two tanks containing liquid and two cubes. The cubes were positioned in front of the tanks. The students were required to pick up each cube by pressing and holding the main button in the middle of the ball interface, and place it in the liquid held by the tank behind it. The material properties of each cube were derived from two sizes (small or large) and two colors (white or gray). The large cube's volume was eight times as large as that of the small cube. In all activities except Activity 3, the white cube represented an object of small mass, while the gray represented a large mass.

The tanks were initialized to contain the same amount of liquid. Fluid properties were denoted by two liquid colors in the tank; blue represented water, and yellow represented maple syrup. Two volume marks were placed on each tank so that the students could observe any changes in the liquid level once a cube was placed in the tank. A blue or yellow mark corresponded to the maximum height that the liquid level could be raised when a large or small cube was fully submerged, respectively. In the rest of this section, we describe the visual and haptic stimuli presented to the students during each of the five activities.

In Activity 1, V and $\mathrm{VH}$ students were presented with a screen containing two white cubes: one large and one small. Each cube was placed in front of a tank containing water (see Fig. 2). Students were asked to observe any changes in the water level as 
they held each cube at and below the water surface. They were then asked to assess which cube had a larger volume by observing which cube experienced greater water displacement when it was pushed down to be fully submerged. In addition to the visual stimuli, the VH students also felt the change in forces exerted on each cube as it was lowered into the water tank.

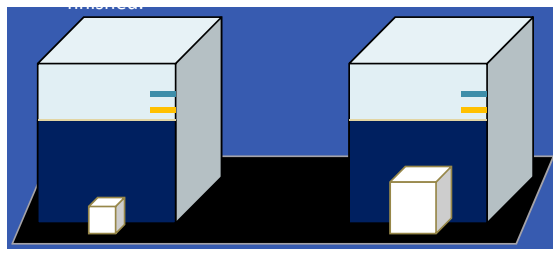

Figure 2. A screenshot of Activity 1 - Volume

The participants studied the concept of volume during Activity 2. The computer screen showed two cubes of identical volume, one gray and one white, in front of two identical water tanks (see Fig. 3). The students were asked to determine which of the two cubes had the greater mass. Visually, the students observed that the gray cube sank to the bottom of the water tank while the white cube floated in the water tank. VH students were again able to feel the force changes as they placed each cube into the tank. This activity was designed to show the students that two cubes of the same volume behaved differently when placed in a liquid, due to the difference in the cubes' mass (weight). At this point, buoyancy was defined to the students as "the tendency for an object to float or sink."

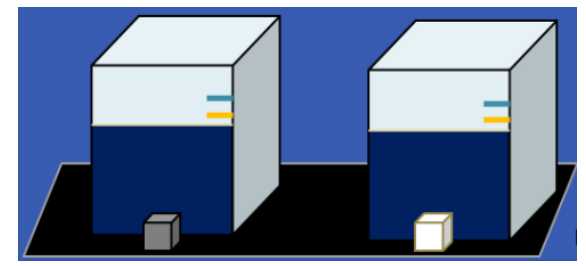

Figure 3. A screenshot of Activity 2 - Mass

The participants studied the concept of density in Activity 3, in which the students were presented with two identical-looking white cubes in front of two identical water tanks (see Fig. 4). The mass of one of the cubes was greater than that of the other cube. The cube with the greater mass sank in the water tank while the other cube floated. Students were asked to determine which of the two cubes had a greater density. While the V students could only observe the sinking/floating behavior of the two cubes, the $\mathrm{VH}$ students also felt each cube's weight as it was picked up and the change in the total force exerted on each cube as it was submerged into the water tank.

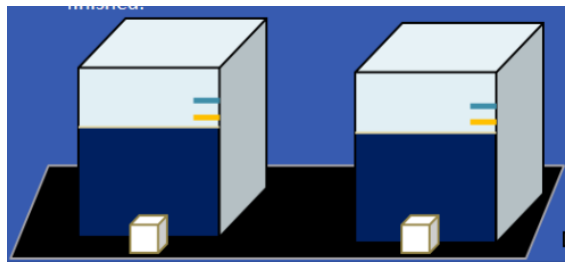

Figure 4. A screenshot of Activity 3 - Density

Floating and sinking behavior was described to the students as an effect caused by the balance between the (upward) buoyant force and the (downward) weight force acting on the cube. Students observed this effect further in Activities 4 and 5 (see Fig.
5), in which they were shown that the fluid surrounding a cube can change the cube's ability to float or sink. In Activity 4 (Fig. 5a), the students observed that the white cube in the water tank (blue liquid on the left) displaced more liquid than the white cube in the maple syrup tank (yellow liquid on the right). The students were told that the two white cubes had the same material properties, but they could observe that the cube in the water tank submerged more than the one in the maple syrup tank. The students were also told that the maple syrup had a greater density than the water.

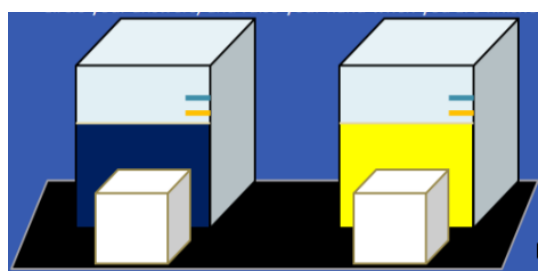

(a) Activity 4

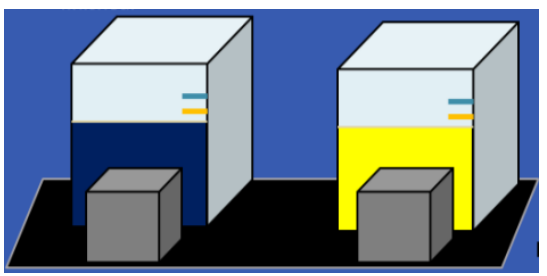

(b) Activity 5

Figure 5. Screenshots of Activities 4 and 5 - Density of surrounding fluid

The effect of fluid density on the net force acting on the cube was demonstrated again in Activity 5 , in which one gray cube sank in water, while the other identical gray cube floated in the maple syrup (see Fig. 5b). Again, V students could only observe the visual trajectory information while VH students could feel the forces as well.

\subsection{Procedures}

Each participant completed a training session to eliminate any performance biases that may result from the user's unfamiliarity with the Falcon force feedback device. The training session was conducted prior to the session in which the students used the buoyancy force simulation. During the training session, students performed a series of tasks within a modified version of the 'ODE Cube' demo taken from the CHAI3D toolkit. The students were presented with three cubes of different colors on the screen (green, pink, and blue). The pink and blue cubes were of the same size. The green cube's volume was eight times that of the pink or blue cube. The blue cube was twice as heavy as the green cube and four times as heavy as the pink cube. All students received the same visual and haptic information for each task in the training session. Students were asked to pick up each cube and make comparisons regarding each cube's weight. They were also asked to stack the cubes in various configurations (e.g. put one on top of another in any order; put the blue and pink cubes next to each other and stack the green cube on top of the adjacent blue and pink cubes, etc.).

The students answered several open-ended questions pertaining to their experience during the training session. Some questions were designed to assess the student's dexterity (e.g., "Try to stack the cubes, one on top of the other. How many tries did it take 
you?"), while others were more conceptual (e.g, "Does the blue cube weigh more than the green cube? If so, why do you think it weighs more?"). The students also completed a pretest of content questions related to buoyancy that evaluated their current knowledge about buoyancy and relevant concepts concerning material properties. The questions in the content test followed similar structures as those found in other assessment tools such as the Force Concept Inventory [15].

The student participants came from one fourth-grade class and three sixth grade classes. For each class, the students were randomly assigned into the visual (V) and visuohaptic (VH) groups based on their pretest scores. Specifically, pretest scores for all participants were scored in terms of the number of correctly-answered questions and ranked from the highest to the lowest for each of the four classes. Starting from highest-scoring student, for every student assigned to the $\mathrm{V}$ group, the student with the next highest score was assigned to the VH group. This continued until all students in the class were assigned to either the $\mathrm{V}$ or VH group. This approach was used in an effort to match, as much as possible, the $\mathrm{V}$ and $\mathrm{VH}$ groups within the same class with respect to their understanding of buoyancy concepts prior to the main study. A total of forty-four VH students and forty-three $\mathrm{V}$ students took part in the study. The same buoyancy simulations were used by students in both groups, except that force output was disabled for the $\mathrm{V}$ group.

The main study on buoyancy was completed in one 40-minute class period for each class. The instruction was subdivided into the four core ideas that have been discussed in other buoyancy curricula studies [13-14] as misleading concepts. The four topics were object volume, object density, object trajectory as described by buoyant and total forces, and surrounding fluid density. Each idea was presented to the participants in a separate module consisting of a PowerPoint presentation, followed by an interactive visual or visuohaptic simulation through which the students could further investigate the concept that had just been presented. The order of the modules was designed such that students could use their intuitive understanding of material properties as a basis of understanding buoyant force as well as the inherent material properties of an object and its surroundings that govern the buoyant force. Participants completed a series of activities associated with each module (see Fig. 6).

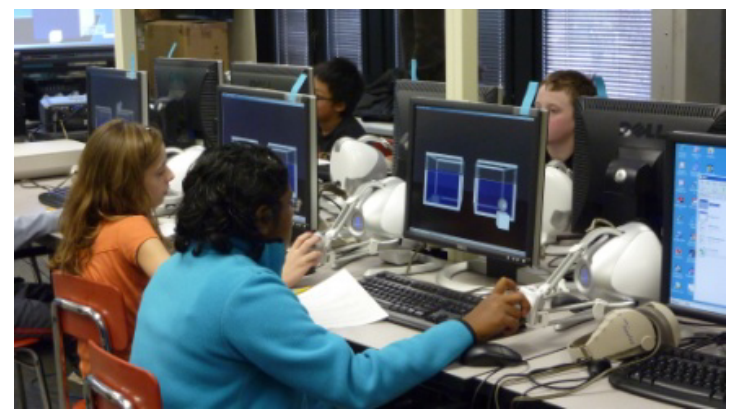

Figure 6. VH students with Falcons during buoyancy lesson

All simulations consisted of a cube to be placed in a tank. A maximum of two cubes and two tanks were presented at any given time if students were asked to make a comparison. After all four modules and the associated five activities were finished, the students completed a posttest of multiple-choice content questions related to buoyancy before they were dismissed. The posttest contained the same questions as the pretest, except that the last question's answer choices were modified slightly.
Each of the questions was designed to highlight one of the four ideas that were presented along with the five activities using visual or visuohaptic simulations. The first four questions assessed students' ability to recognize changes in liquid level as a function of a partially submerged object's material properties (i.e. volume and density) or that of the surrounding fluid. These questions were based from concepts discussed during Activities 1, 2,4 , and 5. An example of such a question is shown in Fig. 7. The next three questions relied on students' understanding of the balance of buoyant and weight forces as they related to the object's and the surrounding fluid's material properties, as highlighted in Activities 2, 3, 4, and 5. One such question is as follows: "When an object sinks, the buoyant force acting on the object is: A) Larger than the object's weight; B) The same as the object's weight; C) Smaller than the object's weight." The final three questions pertained specifically to an object's buoyant force and its relationship to its weight force in the context of floating or sinking behavior. An example is as follows: "You are on a submarine that is fully underwater at the bottom of the sea. To help the submarine rise up to the surface of the water, the captain is going to empty some water from a tank inside the submarine. Why do you think this works? A) It works because the buoyant force will be smaller than the weight force; B) It works because the buoyant force will be larger than the weight force; C) It works because the weight force will be larger than the buoyant force."

\section{Question 3. Look at the figure below:}

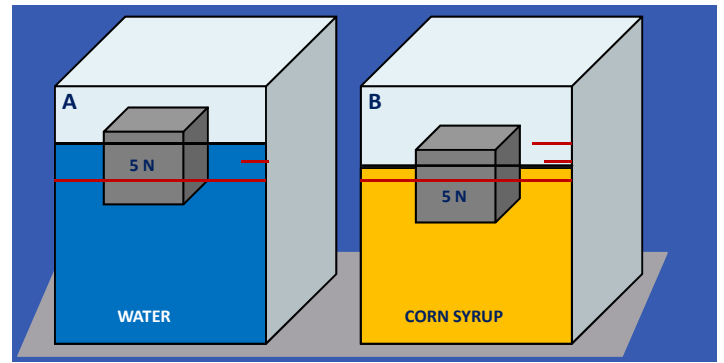

The object in each tank is floating. Which object displaces more liquid?

A) The object in Tank A

B) The object in Tank B

C) The objects in Tank A and B displace the same amount of liquid.

Why does this happen?

A) The object in corn syrup (Tank B) weighs less than the object in water (Tank A).

B) The object weighs the same, but corn syrup is denser than water.

C) The object in corn syrup (Tank B) weighs more than the object in water (Tank $A$ ).

Figure 7. An example multiple-choice question from post-test

\subsection{Data Analysis}

The independent variables of the present study were grade level (fourth and sixth) and experimental condition (V vs. VH). The dependent variables were the pretest and posttest scores in terms of the number of correctly-answered questions out of the 10 content questions.

The data were pooled across groupings and within groupings to assess the normality of the data sets for the purpose of determining which statistical tests could be used to analyze the data. The score distributions were skewed, requiring the use of nonparametric tests for comparisons. The Wilcoxon-MannWhitney rank-sum test was used to examine and compare the 


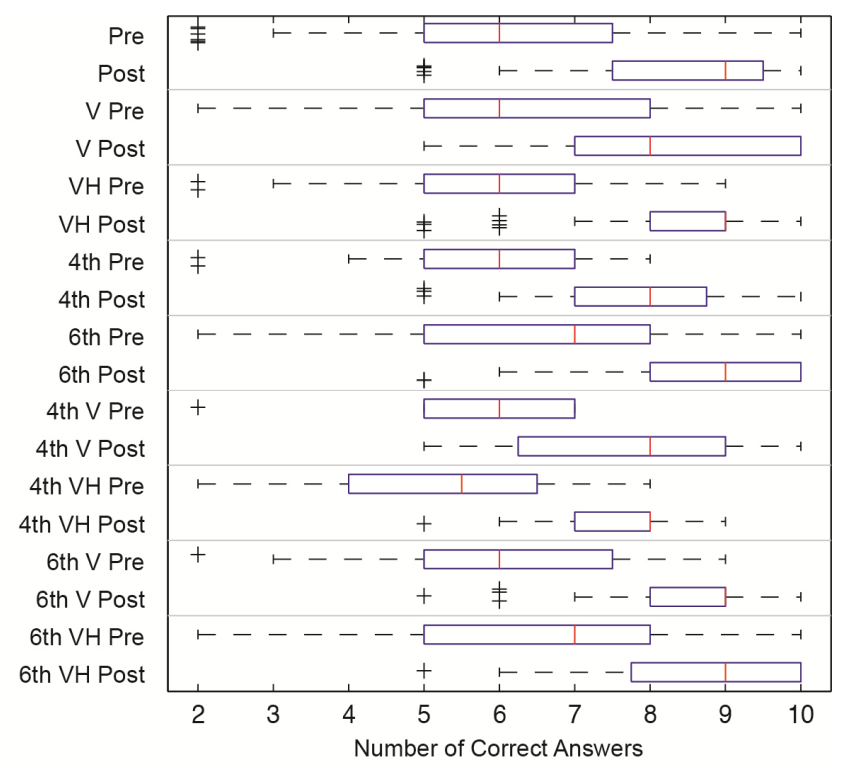

Figure 8. Boxplot of pretest and posttest scores by grade level and experimental condition.

distributions of posttest and pretest scores (see [16-17] for why, when and how to use the rank sum test). The procedure does not assume a normal distribution, handles unbalanced data, and is robust when outliers exist because observations are replaced by rankings. The method requires similar distribution types for comparisons and assumes approximate equality of variance, which is typically measured as the interquartile difference of the distribution.

The Wilcoxon signed rank test, the nonparametric analogue of the paired difference t-test, was conducted to gauge the amount of learning achieved. The differences between the medians of pretest and posttest scores for the various groupings were examined. Finally, a rank analysis of covariance (rank ANCOVA) with Cochran-Mantel-Haenszel statistics was conducted to simultaneously study the overall main effects of grade and condition. ANCOVA is a popular method because inclusion of a covariate (pretest score) reduces variability and increases the power of the test. However, it is important to note that for negatively skewed distributions (post-test scores), the WilcoxonMann-Whitney has superior relative efficiency compared to both ANCOVA and the t-test [18]. Further, in the case presented, one grouping, based on grade level, is non-randomized and the distributions of the covariate, pre-test score, differ.

\section{Results}

A 10 question multiple-choice content test was developed by the researchers, one of whom is a middle-school science teacher, to measure student aptitude of buoyancy concepts before and after the presentation of the buoyancy instructional materials and the hands-on activities using the simulations developed for the present study. The results of the tests are shown in Figure 8. Each horizontal box and whisker diagram shows the lower quartile (q1, left edge of box), median (dark red line inside the box), upper quartile (q3, right edge of box), and outliers (the black "+" symbols). The whiskers are drawn to the length of the interquartile range (q3-q1). In general, the posttest scores were higher than the pretest scores for all groups, the sixth graders scored higher than the fourth graders, and the scores for the $\mathrm{V}$ and
Table 1. Comparisons results from the Wilcoxon rank-sum test

\begin{tabular}{|c|c|c|c|r|r|}
\hline$\#$ & Group A & Group B & p value & $\begin{array}{c}\text { Sum } \\
\text { min } \\
\left(\mathbf{R}_{\mathbf{A}}, \mathbf{R}_{\mathbf{B}}\right)\end{array}$ & $\begin{array}{c}\text { Expected } \\
\text { Sum } \\
\text { under } \mathbf{H}_{\mathbf{0}}\end{array}$ \\
\hline 1 & Post & Pre & $<.0001$ & 5440.0 & 7788.0 \\
\hline 2 & V, Post & V, Pre & $<.0001$ & 1286.5 & 1870.5 \\
\hline 3 & VH, Post & VH, Pre & $<.0001$ & 1468.0 & 2047.5 \\
\hline 4 & V, Post & VH, Post & 0.8989 & 1928.5 & 1913.5 \\
\hline 5 & V, Pre & VH, Pre & 0.7474 & 1875.0 & 1913.5 \\
\hline 6 & $4^{\text {th }}$, Post & $6^{\text {th }}$ Post & 0.0061 & 734.5 & 1023.5 \\
\hline 7 & $4^{\text {th }}$, Pre & $6^{\text {th }}$, Pre & 0.0463 & 813.0 & 1023.5 \\
\hline 8 & $4^{\text {th }}$, V, Post & $4^{\text {th }}$, V, Pre & 0.0130 & 89.0 & 126.5 \\
\hline 9 & $4^{\text {th }}, \mathrm{VH}$, Post & $4^{\text {th }}$, VH, Pre & 0.0038 & 100.5 & 150.0 \\
\hline 10 & $6^{\text {th }}$, V, Post & $6^{\text {th }}$, V, Pre & $<.0001$ & 711.5 & 1040.0 \\
\hline 11 & $6^{\text {th }}, \mathrm{VH}$, Post & $6^{\text {th }}, \mathrm{VH}$, Pre & $<.0001$ & 793.5 & 1105.5 \\
\hline
\end{tabular}

VH groups were similar at each grade level and for all students pooled across grade levels.

The Wilcoxon-Rank Sum Test was conducted to compare the distributions with the assumption of equal spreads. The comparisons of interest are shown in Table 1 and Figure 8. All studied groupings illustrated a higher median posttest score relative to the median pretest score. The first entry compared pretest scores to posttest scores, pooled across grade levels and experimental conditions. It showed a significant difference in test scores due to the buoyancy instruction and learning activities. The second and third entries compared the difference in scores achieved by the $\mathrm{V}$ and $\mathrm{VH}$ groups, respectively, pooled across the fourth graders and the sixth graders. The fourth and fifth entries show that the groups were appropriately balanced on the basis of pretest scores and that there was no significant difference in overall posttest or pretest scores, respectively, between the $\mathrm{V}$ and VH groups. Entries 6 and 7 compare the difference between posttest and pretest scores, respectively of 4th and 6th graders. The results indicate that the 6 th graders achieved a higher score on both the pretest and the posttest. Entries 8-11 compared the pretest and posttest scores across four groups $(2$ grade levels $\times 2$ conditions), respectively, and concluded that all groups achieved significantly higher posttest scores than pretest scores.

The Wilcoxon signed rank test was performed on the difference scores (posttest score - pretest score) for various groupings. For entries 1-3 and 8-11 (Table 1) the difference scores were significantly different from zero and each grouping achieved a median positive difference of 2.0 from pretest to posttest (except for the 2.5 achieved by the 4 th grade $\mathrm{VH}$ group) with an interquartile difference of 2.0. The Kruskal-Wallis test, the nonparametric analogue of a one-way ANOVA, was also performed on the difference scores for entries 8-11 $(\mathrm{SS}=70.8$, $\left.\mathrm{df}=3, \chi^{2}=0.11, \mathrm{p}=0.9903\right)$. The results indicated that no matter along which criterion the participants were grouped, they learned similar amounts and derived the same benefits from the computer simulations.

Finally, a rank transform ANCOVA was performed with the dependent variable posttest score and independent categorical variables of grade and condition $(\mathrm{V}, \mathrm{VH})$ and a covariate pretest score. The results were consistent with the Wilcoxon-MannWhitney data shown in Table 1. The Cochran-Mantel-Haenszel $(\mathrm{CMH})$ statistic for the $\mathrm{V}$ versus $\mathrm{VH}$ grouping showed that these conditions were not significantly different when controlling for grade $\left(\mathrm{CMH} \chi^{2}=.0066, \mathrm{p}=.9353\right)$. The statistic showed that posttest scores for the $4^{\text {th }}$ versus $6^{\text {th }}$ grade groupings were significantly different when controlling for condition (CMH $\chi^{2}=5.6793, \mathrm{p}=.0172$ ). 


\section{Discussion}

In the present study, we developed instructional materials and visuohaptic computer simulations for teaching buoyant forces at the elementary school level. Compared to visual simulations that typically convey force information via object trajectory, velocity and acceleration, visuohaptic simulations allow users to feel force changes directly through a force-feedback device. We hypothesized that the additional force information provided by a force-feedback device may contribute to a better understanding of concepts related to buoyant force. Our results, however, did not support the expectation. We found that students in both the visual group and the visuohaptic group improved their understanding of buoyancy as shown by the significant increase in test scores from pretest to posttest, but there was no demonstrable significant effect due to haptic feedback. There are two possible reasons for the result. First, the Falcon force-feedback device is limited in force output range and its performance varies from unit to unit. Compromises had to be made in the selection of simulation parameters in order to maintain device stability and not to exceed its maximum output force. For example, it was difficult to increase the differences between the densities of the two fluids, or the masses of the two cubes to improve their discriminability. The Falcon was selected for the present study mainly for its affordability for use in an elementary school setting. It is unclear to what extent the limitation of the Falcon precluded the demonstration of additional benefits provided by force information in visuohaptic simulations. Second, the questions in the content test were developed mainly to gauge student learning of buoyancy concepts. In hindsight, many of the questions in our content test were more visually-based (e.g., asking students to compare the amount of fluid displaced by two cubes) than visuohaptic- or haptically-based (e.g., "Which of the two cubes was heavier in the water?"). Such force-oriented questions may help tease out concepts that are especially well conveyed by force feedback.

One interesting and important finding of the present study was that the fourth-grade students improved as much as the sixthgrade students from pretest to posttest, indicating that the younger students were able to learn as much as the older students. This suggests the efficacy of using computer-based simulations in science classrooms. It would be interesting to see if the buoyancy concepts can be effectively taught at even younger ages using the visuohaptic simulations we have developed for the present study, since visual or visuohaptic simulations may be especially useful in engaging young students and enhancing their learning.

To continue research on when and how haptic feedback may contribute to better learning, we need higher-quality force feedback devices that are still affordable. Designing instructional and assessment materials that focus more on the haptic modality is also of a high priority. Ultimately, we need to design studies that not only investigate students' comprehension of force concepts, but measure the transfer of learning that may be attributable to visuohaptic simulations. We plan to continue to work closely with science teachers in developing visuohaptic simulations of concepts that are in the science curriculum for elementary schools.

\section{ACKNOWLEDGMENT}

The authors thank Mrs. Katie Dodds at the Happy Hollow Elementary School for her assistance with the study. This material is based upon work supported by the National Science Foundation under Grant No. EEC-0836664 (a Graduate Research Supplement Award for C.S.), No. DRL-1043026 (for J.J.Y., H.Z.T. and J.C.), and No. EEC-0943177 (for H.Z.T., B.D. and G.B.). J.C. was also supported through an Explora Pro Grant from Région Rhône Alpes.

\section{REFERENCES}

[1] A. M. Okamura, C. Richard, and M. R. Cutkosky, "Feeling is believing: Using a force-feedback joystick to teach dynamic systems," Journal of Engineering Education 92, 345-349, 2002.

[2] F. P. J. Brooks, M. Ouh-Young, J. J. Batter, and P. J. Kilpatrick, "Project GROPE - haptic displays for scientific visualization," Computer Graphics 24, 177-185, 1990.

[3] M. G. Jones, T. Andre, R. Superfine, and R. Taylor, "Learning at the nanoscale: The impact of students' use of remote microscopy on concepts of viruses, scale, and microscopy," Journal of Research in Science Teaching 40, 303-322, 2003.

[4] J. Park, K. Kim, H. Z. Tan, R. Reifenberger, G. Bertoline, T. Hoberman, and D. Bennett, "An initial study of visuohaptic simulation of point-charge interactions," Proceedings of the Symposium on Haptic Interfaces for Virtual Environments and Teleoperator Systems, 425-430, 2010.

[5] F. G. Hamza-Lup and M. Adams, "Feel the pressure:E-learning system with haptic feedback," Proceedings of the Symposium on Haptic Interfaces for Virtual Environments and Teleoperator Systems, 445-450, 2008.

[6] M. Reiner, "Conceptual construction of fields through tactile interface," Interactive Learning Environments 7, 31-55, 1999.

[7] G. Millet, A. Lécuyer, J. M. Burkhardt, D. S. Haliyo, and S. Régnier, "Improving perception and understanding of nanoscale phenomena using haptics and visual analogy," Proceedings of EuroHaptics 2008, 847-856, 2008

[8] J. Minogue and M. G. Jones, "Haptics in education: Exploring an untapped sensory modality," Review of Educational Research 76, 317-348, 2006.

[9] C. R. Wagner, N. Stylopoulos, P. G. Jackson, and R. D. Howe, "The benefit of force feedback in surgery: Examination of blunt dissection," Presence: Teleoperators and Virtual Environments 16, 252-262, 2007.

[10] S. Baillie, A. Crossan, S. Brewster, D. Mellor, and S. Reid, "Validation of a bovine rectal palpation simulator for training veterinary students," Studies in Health Technology \& Informatics 111, 33-36, 2005.

[11] Y. Li, V. Patoglu, and M. O'Malley, "Shared Control for Training in Virtual Environments: Learning Through Demonstration?," Proceedings of EuroHaptics 2006.

[12] D. Morris, H. Z. Tan, F. Barbagli, T. Chang, and K. Salisbury, "Haptic feedback enhances force skill learning," Proceedings of the World Haptics Conference, 21-26, 2007.

[13] P. R. L. Heron, M. E. Loverude, P. S. Shaffer, and L. C. McDermott, "Helping students develop an understanding of Archimedes' principle: II. Development of research-based instructional materials," American Journal of Physics 71, 1188-1195, 2003.

[14] M. E. Loverude, C. H. Kautz, and P. R. L. Heron, "Helping students develop an understanding of Archimedes' principle: I. Research on student understanding," American Journal of Physics 71, 1178-1187, 2003.

[15] D. Hestenes, M. Wells, and G. Swackhamer, "Force concept inventory," The Physics Teacher 30, 141-158, 1992.

[16] L. M. LaVange and G. G. Koch, "Rank score tests," Circulation 114, 2528-2533, 2006.

[17] M. Fay and M. A. Proschan, "Wilcoxon-Mann-Whitney or t-test? On assumptions for hypothesis tests and multiple interpretations of decision rules," Statistics Surveys 4, 1-39, 2010.

[18] A. J. Vickers, "Parametric versus non-parametric statistics in the analysis of randomized trials with non-normally distributed data," BMC Medical Research Methodology 5:35, 2005 